\title{
On a more accurate half-discrete mulholland's inequality and an extension
}

\author{
Qiang Chen ${ }^{1}$ and Bicheng Yang ${ }^{2^{*}}$
}

* Correspondence: bcyang@gdei. edu.cn

${ }^{2}$ Department of Mathematics, Guangdong University of

Education, Guangzhou, Guangdong 510303, PR China

Full list of author information is available at the end of the article

\section{Abstract}

By using the way of weight functions and Jensen-Hadamard's inequality, a more accurate half-discrete Mulholland's inequality with a best constant factor is given. The extension with multi-parameters, the equivalent forms as well as the operator expressions are considered.

Mathematics Subject Classication 2000: 26D15; 47A07.

Keywords: Mulholland's inequality, weight function, equivalent form, operator expression

\section{Introduction}

Assuming that $f, g \in L^{2}\left(R_{+}\right),\|f\|=\left\{\int_{0}^{\infty} f^{2}(x) d x\right\}^{\frac{1}{2}}<0,\|g\|<0$, we have the following Hilbert's integral inequality (cf. [1]):

$$
\int_{0}^{\infty} \int_{0}^{\infty} \frac{f(x) g(y)}{x+y} d x d y<\pi\|f\|\|g\|
$$

where the constant factor $\pi$ is the best possible. Moreover, for $a=\left\{a_{m}\right\}_{m=1}^{\infty} \in l^{2}, b=\left\{b_{n}\right\}_{n=1}^{\infty} \in l^{2},\|a\|=\left\{\sum_{m=1}^{\infty} a_{m}^{2}\right\}^{\frac{1}{2}}<0,\|b\|>0$, we still have the following discrete Hilbert's inequality

$$
\sum_{m=1}^{\infty} \sum_{n=1}^{\infty} \frac{a_{m} b_{n}}{m+n}<\pi\|a\|\|b\|
$$

with the same best constant factor $\pi$. Inequalities (1) and (2) are important in analysis and its applications (cf. [2-4]) and they still represent the field of interest to numerous mathematicians. Also we have the following Mulholland's inequality with the same best constant factor (cf. [1,5]):

$$
\sum_{m=2}^{\infty} \sum_{n=2}^{\infty} \frac{a_{m} b_{n}}{\ln m n}<\pi\left\{\sum_{m=2}^{\infty} m a_{m}^{2} \sum_{n=2}^{\infty} n b_{n}^{2}\right\}^{\frac{1}{2}}
$$

\section{Springer}

(c) 2012 Chen and Yang; licensee Springer. This is an Open Access article distributed under the terms of the Creative Commons Attribution License (http://creativecommons.org/licenses/by/2.0), which permits unrestricted use, distribution, and reproduction in any medium, provided the original work is properly cited. 
In 1998, by introducing an independent parameter $\lambda \in(0,1]$, Yang [6] gave an extension of (1). By generalizing the results from [6], Yang [7] gave some best extensions of (1) and (2) as follows: If $p>1, \frac{1}{p}+\frac{1}{q}=1, \lambda_{1}+\lambda_{2}=\lambda, k_{\lambda}(x, y)$ is a non-negative homogeneous function of degree $-\lambda$ satisfying $k\left(\lambda_{1}\right)=\int_{0}^{\infty} k_{\lambda}(t, 1) t^{\lambda_{1}-1} d t \in R_{+}$, $\phi(x)=x^{p\left(1-\lambda_{1}\right)-1}, \psi(x)=x^{q\left(1-\lambda_{2}\right)-1}, f(\geq 0) \in L_{p, \phi}\left(R_{+}\right)=\left\{f\|f\|_{p, \phi}:=\left\{\int_{0}^{\infty} \phi(x)|f(x)|^{p} d x\right\}^{\frac{1}{p}}<\infty\right\}, g(\geq 0) \in L_{q, \psi}\left(R_{+}\right),\|f\|_{p, \phi}\|\delta\|_{q, \psi}>0$ then

$$
\int_{0}^{\infty} \int_{0}^{\infty} k_{\lambda}(x, y) f(x) g(y) d x d y<k\left(\lambda_{1}\right)\|f\|_{p, \phi}\|g\|_{q, \psi^{\prime}}
$$

where the constant factor $k\left(\lambda_{1}\right)$ is the best possible. Moreover if $k_{\lambda}(x, y)$ is finite and $k_{\lambda}(x, y) x^{\lambda_{1}-1}\left(k_{\lambda}(x, y) \gamma^{\lambda_{2}-1}\right)$ is decreasing for $x>0(y>0)$, then for $a=\left\{a_{m}\right\}_{m=1}^{\infty} \in l_{p, \phi}:=\left\{a \mid\|a\|_{p, \phi}:=\left\{\sum_{n=1}^{\infty} \phi(n)\left|a_{n}\right|^{p}\right\}^{\frac{1}{p}}<\infty\right\}, b=\left\{b_{n}\right\}_{n=1}^{\infty} \in l_{q, \psi},\|a\|_{p, \phi}\|b\|_{q, \psi}>0$ we have

$$
\sum_{m=1}^{\infty} \sum_{n=1}^{\infty} k_{\lambda}(m, n) a_{m} b_{n}<k\left(\lambda_{1}\right)\|a\|_{p, \phi}\|b\|_{q, \psi}
$$

where, $k\left(\lambda_{1}\right)$ is still the best value. Clearly, for $p=q=2, \lambda=1, k_{1}(x, y)=\frac{1}{x+y}, \lambda_{1}=\lambda_{2}=\frac{1}{2}$, inequality (4) reduces to (1), while (5) reduces to (2). Some other results about Hilbert-type inequalities are provided by [8-16].

On half-discrete Hilbert-type inequalities with the general non-homogeneous kernels, Hardy et al. provided a few results in Theorem 351 of [1]. But they did not prove that the the constant factors in the inequalities are the best possible. However Yang [17] gave a result with the kernel $\frac{1}{(1+n x)^{\lambda}}$ by introducing an interval variable and proved that the constant factor is the best possible. Recently, Yang [18] gave the following half-discrete Hilbert's inequality with the best constant factor $B\left(\lambda_{1}, \lambda_{2}\right)\left(\lambda_{1}>0,0<\lambda_{2}\right.$ $\left.\leq 1, \lambda_{1}+\lambda_{2}=\lambda\right)$ :

$$
\int_{0}^{\infty} f(x) \sum_{n=1}^{\infty} \frac{a_{n}}{(x+n)^{\lambda}} d x<B\left(\lambda_{1}, \lambda_{2}\right)\|f\|_{p, \phi}\|a\|_{q, \psi} .
$$

In this article, by using the way of weight functions and Jensen-Hadamard's inequality, a more accurate half-discrete Mulholland's inequality with a best constant factor similar to (6) is given as follows:

$$
\int_{\frac{3}{2}}^{\infty} f(x) \sum_{n=2}^{\infty} \frac{a_{n}}{\ln \frac{4}{9} x n} d x<\pi\left\{\int_{\frac{3}{2}}^{\infty} x f^{2}(x) d x \sum_{n=2}^{\infty} n a_{n}^{2}\right\}^{\frac{1}{2}} .
$$


Moreover, a best extension of (7) with multi-parameters, some equivalent forms as well as the operator expressions are also considered.

\section{Some lemmas}

Lemma 1 If $\lambda_{1}>0,0<\lambda_{2} \leq 1, \lambda_{1}+\lambda_{2}=\lambda, \alpha \geq \frac{4}{9}$, setting weight functions $\omega(n)$ and $\varpi(x)$ as follows:

$$
\begin{gathered}
\omega(n):=(\ln \sqrt{\alpha} n)^{\lambda_{2}} \int_{\frac{1}{\sqrt{\alpha}}}^{\infty} \frac{(\ln \sqrt{\alpha} x)^{\lambda_{1}-1}}{x(\ln \alpha x n)^{\lambda}} d x, \quad n \in \mathbf{N} \backslash\{1\}, \\
\varpi(x):=(\ln \sqrt{\alpha} x)^{\lambda_{1}} \sum_{n=2}^{\infty} \frac{(\ln \sqrt{\alpha} n)^{\lambda_{2}-1}}{n(\ln \alpha x n)^{\lambda}}, \quad x \in\left(\frac{1}{\sqrt{\alpha}}, \infty\right),
\end{gathered}
$$

then we have

$$
\varpi(x)<\omega(n)=B\left(\lambda_{1}, \lambda_{2}\right) .
$$

Proof. Applying the substitution $t=\frac{\ln \sqrt{\alpha} x}{\ln \sqrt{\alpha} n}$ to (8), we obtain

$$
\omega(n)=\int_{0}^{\infty} \frac{1}{(1+t)^{\lambda}} t^{\lambda_{1}-1} d t=B\left(\lambda_{1}, \lambda_{2}\right) .
$$

Since by the conditions and for fixed $x \geq \frac{1}{\sqrt{\alpha}}$,

$$
h(x, y):=\frac{(\ln \sqrt{\alpha} y)^{\lambda_{2}-1}}{y(\ln \alpha x y)^{\lambda}}=\frac{1}{y(\ln \sqrt{\alpha} x+\ln \sqrt{\alpha} y)^{\lambda}(\ln \sqrt{\alpha} y)^{1-\lambda_{2}}}
$$

is decreasing and strictly convex in $y \in\left(\frac{3}{2}, \infty\right)$, then by Jensen-Hadamard's inequality (cf. [1]), we find

$$
\begin{aligned}
& \varpi(x)<(\sqrt{\alpha} \ln x)^{\lambda_{1}} \int_{\frac{3}{2}}^{\infty} \frac{1}{y(\ln \alpha x y)^{\lambda}}(\ln \sqrt{\alpha} y)^{\lambda_{2}-1} d y \\
& t=(\ln \sqrt{\alpha} y) /(\ln \sqrt{\alpha} x) \int_{=}^{\infty} \frac{t^{\lambda_{2}-1} d t}{(1+t)^{\lambda}} \leq B\left(\lambda_{2}, \lambda_{1}\right) B\left(\lambda_{1}, \lambda_{2}\right), \\
& \frac{\ln (3 \sqrt{\alpha} / 2)}{\ln \sqrt{\alpha} x}
\end{aligned}
$$

namely, (10) follows. $\square \cdot$

Lemma 2 Let the assumptions of Lemma 1 be fulfilled and additionally, $p>1, \frac{1}{p}+\frac{1}{q}=1, a_{n} \geq 0, \in \mathbf{N} \backslash\{1\}, f(x)$ is a non-negative measurable function in $\left(\frac{1}{\sqrt{\alpha}}, \infty\right)$. Then we have the following inequalities: 


$$
\begin{aligned}
\leq & {\left[B\left(\lambda_{1}, \lambda_{2}\right)\right]^{\frac{1}{q}}\left\{\int_{\frac{1}{\sqrt{\alpha}}}^{\infty} \varpi(x) x^{p-1}(\ln \sqrt{\alpha} x)^{p\left(1-\lambda_{1}\right)} f^{p}(x) d x\right\}^{\frac{1}{p}}, } \\
L_{1} & =\left\{\int_{\left.\frac{1}{\sqrt{\alpha}} \frac{(\ln \sqrt{\alpha} x)^{q \lambda_{1}-1}}{x[\varpi(x)]^{q-1}}\left[\sum_{n=2}^{\infty} \frac{a_{n}}{(\ln \alpha x n)^{\lambda}}\right]^{q} d x\right\}^{\frac{1}{q}}}\right\}^{\infty} \\
\leq & \left\{B\left(\lambda_{1}, \lambda_{2}\right) \sum_{n=2}^{\infty} n^{q-1}(\ln \sqrt{\alpha} n)^{q\left(1-\lambda_{2}\right)-1} a_{n}^{q}\right\}^{\frac{1}{q}} .
\end{aligned}
$$

Proof. By Hälder's inequality cf. [1] and (10), it follows

$$
\begin{aligned}
& {\left[\int_{\frac{1}{\sqrt{\alpha}}}^{\infty} \frac{f(x) d x}{(\ln \alpha x n)^{\lambda}}\right]^{p}=\left\{\int_{\frac{1}{\sqrt{\alpha}}}^{\infty} \frac{1}{(\ln \alpha x n)^{\lambda}}\left[\frac{(\ln \sqrt{\alpha} x)^{\left(1-\lambda_{1}\right) / q} x^{1 / q}}{(\ln \sqrt{\alpha} n)^{\left(1-\lambda_{2}\right) / p} n^{1 / p}} f(x)\right]\right.} \\
& \left.\times\left[\frac{\left(\ln \sqrt{\alpha} n^{\left(1-\lambda_{2}\right) / p} n^{1 / p}\right)}{\left(\ln \sqrt{\alpha} x^{\left(1-\lambda_{1}\right) / q} x^{1 / q}\right)}\right] d x\right\}^{p} \leq \int_{\frac{1}{\sqrt{\alpha}}}^{\infty} \frac{x^{p-1}}{(\ln \alpha x n)^{\lambda}} \frac{(\ln \sqrt{\alpha} x)^{\left(1-\lambda_{1}\right)(p-1)}}{n(\ln \sqrt{\alpha} n)^{1-\lambda_{2}}} f^{p}(x) d x \\
& \times\left\{\int_{\frac{1}{\sqrt{\alpha}}}^{\infty} \frac{1}{(\ln \alpha x n)^{\lambda}} \frac{n^{q-1}(\ln \sqrt{\alpha} n)^{\left(1-\lambda_{2}\right)(q-1)}}{x(\ln \sqrt{\alpha} x)^{1-\lambda_{1}}} d x\right\}^{p-1} \\
& =\left\{\omega(n) \frac{(\ln \sqrt{\alpha} n)^{q\left(1-\lambda_{2}\right)-1}}{n^{1-q}}\right\}^{p-1} \int_{\frac{1}{\sqrt{\alpha}}}^{\infty} \frac{x^{p-1}(\ln \sqrt{\alpha} x)^{\left(1-\lambda_{1}\right)(p-1)}}{n(\ln \alpha x n)^{\lambda}(\ln \sqrt{\alpha} n)^{1-\lambda_{2}}} f^{p}(x) d x \\
& =\frac{\left[B\left(\lambda_{1}, \lambda_{2}\right)\right]^{p-1} n}{(\ln \sqrt{\alpha} n)^{p \lambda_{2}-1}} \int_{\frac{1}{\sqrt{\alpha}}}^{\infty} \frac{x^{p-1}(\ln \sqrt{\alpha} x)^{\left(1-\lambda_{1}\right)(p-1)}}{n(\ln \sqrt{\alpha} x n)^{\lambda}(\ln \sqrt{\alpha} n)^{1-\lambda_{2}}} f^{p}(x) d x .
\end{aligned}
$$


Then by Beppo Levi's theorem (cf. [19]), we have

$$
\begin{gathered}
J \leq\left[B\left(\lambda_{1}, \lambda_{2}\right)\right] \frac{1}{q}\left\{\sum_{n=2}^{\infty} \frac{1}{\sqrt{\alpha}} \frac{x^{p-1}(\ln \sqrt{\alpha} x)^{\left(1-\lambda_{1}\right)(p-1)}}{n(\ln \alpha x n)^{\lambda}(\ln \sqrt{\alpha} n)^{1-\lambda_{2}}} f^{p}(x) d x\right\}^{\frac{1}{p}} \\
=\left[B\left(\lambda_{1}, \lambda_{2}\right)\right]^{\frac{1}{q}}\left\{\int_{\frac{1}{\sqrt{\alpha}}}^{\infty} \sum_{n=2}^{\infty} \frac{x^{p-1}(\ln \sqrt{\alpha} x)^{\left(1-\lambda_{1}\right)(p-1)}}{n(\ln \alpha x n)^{\lambda}(\ln \sqrt{\alpha} n)^{1-\lambda_{2}}} f^{p}(x) d x\right\}^{\frac{1}{p}} \\
=\left[B\left(\lambda_{1}, \lambda_{2}\right)\right]^{\frac{1}{q}}\left\{\int_{\frac{1}{\sqrt{\alpha}}}^{\infty} \varpi(x) x^{p-1}(\ln \sqrt{\alpha} x)^{p\left(1-\lambda_{1}\right)-1} f^{p}(x) d x\right\}^{\frac{1}{p}},
\end{gathered}
$$

that is, (11) follows. Still by Hölder's inequality, we have

$$
\begin{aligned}
& {\left[\sum_{n=2}^{\infty} \frac{a}{(\ln \alpha x n)^{\lambda}}\right]^{q}=\left\{\sum_{n=2}^{\infty} \frac{1}{(\ln \alpha x n)^{\lambda}}\left[\frac{(\ln \sqrt{\alpha} x)^{\left(1-\lambda_{1}\right) / q} x^{1 / q}}{(\ln \sqrt{\alpha} n)^{\left(1-\lambda_{2}\right) / p} n^{1 / p}}\right]\right.} \\
\times & {\left.\left[\frac{(\ln \sqrt{\alpha} n)^{\left(1-\lambda_{2}\right) / p} n^{1 / p}}{(\ln \sqrt{\alpha} x)^{\left(1-\lambda_{1}\right) / q} x^{1 / p}} a_{n}\right]\right\}^{q} \leq\left\{\sum_{n=2}^{\infty} \frac{x^{p-1}}{(\ln \alpha x n)^{\lambda}} \frac{(\ln \sqrt{\alpha} x)^{\left(1-\lambda_{1}\right)(p-1)}}{n(\ln \sqrt{\alpha} n)^{1-\lambda_{2}}}\right\}^{q-1} } \\
& \times \sum_{n=2}^{\infty} \frac{1}{(\ln \alpha x n)^{\lambda}} \frac{n^{q-1}(\ln \sqrt{\alpha} n)^{\left(1-\lambda_{2}\right)(q-1)}}{x(\ln \sqrt{\alpha} x)^{1-\lambda_{1}}} a_{n}^{q} \\
= & \frac{x[\varpi(x)]^{q-1}}{(\ln \sqrt{\alpha} x)^{q \lambda_{1}-1}} \sum_{n=2}^{\infty} \frac{(\ln \sqrt{\alpha} x)^{\lambda_{1}-1}}{x(\ln \alpha x n)^{\lambda}} n^{q-1}(\ln \sqrt{\alpha} n)^{(q-1)\left(1-\lambda_{2}\right)} a_{n}^{q} .
\end{aligned}
$$

Then by Beppo Levi's theorem, we have

$$
\begin{aligned}
& L_{1} \leq\left\{\int_{\frac{1}{\sqrt{\alpha}}}^{\infty} \sum_{n=2}^{\infty} \frac{(\ln \sqrt{\alpha} x)^{\lambda_{1}-1}}{x(\ln \alpha x n)^{\lambda}} n^{q-1}(\ln \sqrt{\alpha} n)^{(q-1)\left(1-\lambda_{2}\right)} a_{n}^{q} d x\right\}^{\frac{1}{q}} \\
& =\left\{\sum_{n=2}^{\infty}\left[\begin{array}{c}
(\ln \sqrt{\alpha} n)^{\lambda_{2}} \int_{1}^{\infty} \frac{(\ln \sqrt{\alpha} x)^{\lambda_{1}-1}}{x(\ln \alpha x n)^{\lambda}} d x \\
\left.\frac{\sqrt{\alpha}}{{ }^{2}}\right] n^{q-1}(\ln \sqrt{\alpha} n)^{q\left(1-\lambda_{2}\right)-1} a_{n}^{q}
\end{array}\right\}^{\frac{1}{q}}\right. \\
& =\left\{\sum_{n=2}^{\infty} \omega(n) n^{q-1}(\ln \sqrt{\alpha} n)^{q\left(1-\lambda_{2}\right)-1} a_{n}^{q}\right\}^{\frac{1}{q}},
\end{aligned}
$$

and then in view of (10), inequality (12) follows. $\square$ • 


\section{Main results}

We introduce two functions

$$
\begin{aligned}
& \Phi(x):=x^{p-1}(\ln \sqrt{\alpha} x)^{p\left(1-\lambda_{1}\right)-1}\left(x \in\left(\frac{1}{\sqrt{\alpha}}, \infty\right)\right), \text { and } \\
& \Psi(n):=n^{q-1}(\ln \sqrt{\alpha} n)^{q\left(1-\lambda_{2}\right)-1}(n \in \mathbf{N} \backslash\{1\}),
\end{aligned}
$$

wherefrom, $[\Phi(x)]^{1-q}=\frac{1}{x}(\ln \sqrt{\alpha} x)^{q \lambda_{1}-1}$, and $[\Psi(n)]^{1-p}=\frac{1}{n}(\ln \sqrt{\alpha} n)^{p \lambda_{2}-1}$.

Theorem 3

$p>1, \frac{1}{p}+\frac{1}{q}=1, \lambda_{1}>0,0<\lambda_{2} \leq 1, \lambda_{1}+\lambda_{2}=\lambda, \alpha \geq \frac{4}{9}, f(x), a_{n} \geq 0, f \in L_{p, \Phi}\left(\frac{1}{\sqrt{\alpha}}, \infty\right), a=\left\{a_{n}\right\}_{n=2}^{\infty} \in l_{q, \psi},\|f\|_{p, \Phi}>0$, then we have the following equivalent inequalities:

$$
\begin{aligned}
& I:=\sum_{n=2}^{\infty} \frac{\int_{\frac{1}{\sqrt{\alpha}}}^{\infty}}{(\ln \alpha x n)^{\lambda}}=\int_{\frac{1}{\sqrt{\alpha}}}^{\infty} \sum_{n=2}^{\infty} \frac{f(x) a_{n} d x}{(\ln \alpha x n)^{\lambda}}<B\left(\lambda_{1}, \lambda_{2}\right)\|f\|_{p, \Phi}\|a\|_{q, \Psi}, \\
& J=\left\{\sum_{n=2}^{\infty}[\Psi(n)]^{1-p}\left[\int_{\frac{1}{\sqrt{\alpha}}}^{\infty} \frac{f(x) d x}{(\ln \alpha x n)^{\lambda}}\right]^{p}\right\}^{\frac{1}{p}}<B\left(\lambda_{1}, \lambda_{2}\right)\|f\|_{p, \Phi^{\prime}} \\
& L:=\left\{\int_{\frac{1}{\sqrt{\alpha}}}^{\infty}[\Phi(x)]^{1-q}\left[\sum_{n=2}^{\infty} \frac{a_{n}}{(\ln \alpha x n)^{\lambda}}\right]^{q} d x\right\}^{\frac{1}{q}}<B\left(\lambda_{1}, \lambda_{2}\right)\|a\|_{q, \Psi},
\end{aligned}
$$

where the constant $B\left(\lambda_{1}, \lambda_{2}\right)$ is the best possible in the above inequalities.

Proof. By Beppo Levi's theorem (cf. [19]), there are two expressions for $I$ in (13). In view of (11), for $\varpi(x)<B\left(\lambda_{1}, \lambda_{2}\right)$, we have (14). By Hälder's inequality, we have

$$
I=\sum_{n=2}^{\infty}\left[\Psi_{\frac{1}{\sqrt{\alpha}}}^{\frac{-1}{q}}(n) \int^{\infty} \frac{1}{(\ln \alpha x n)^{\lambda}} f(x) d x\right]\left[\Psi^{\frac{1}{q}}(n) a^{n}\right] \leq J\|a\|_{q, \Psi} .
$$

Then by (14), we have (13). On the other-hand, assuming that (13) is valid, setting

$$
a_{n}:=[\Psi(n)]^{1-p}\left[\int_{\frac{1}{\sqrt{\alpha}}}^{\infty} \frac{1}{(\ln \alpha x n)^{\lambda}} f(x) d x\right]^{p-1}, n \in \mathbf{N} \backslash\{1\},
$$


then $J^{p-1}=\|a\|_{q}, \Psi$. By (11), we find $J<\alpha$. If $J=0$, then (14) is valid trivially; if $J>0$, then by (13), we have

$$
\begin{aligned}
& \|a\|_{q, \Psi}^{q}=J^{p}=I<B\left(\lambda_{1}, \lambda_{2}\right)\|f\|_{p, \Psi}\|a\|_{q, \Psi}, \text { i.e. } \\
& \|a\|_{q, \Psi}^{q-1}=J<B\left(\lambda_{1}, \lambda_{2}\right)\|f\|_{p, \Phi^{\prime}}
\end{aligned}
$$

that is, (14) is equivalent to (13). By (12), since $[\varpi(x)]^{1-q}>\left[B\left(\lambda_{1}, \lambda_{2}\right)\right]^{1-} q$, we have (15). By Hälder's inequality, we find

$$
I=\int_{\frac{1}{\sqrt{\alpha}}}^{\infty}\left[\Phi^{\frac{1}{p}}(x) f(x)\right]\left[\Phi^{\frac{-1}{p}}(x) \sum_{n=2}^{\infty} \frac{a_{n}}{(\ln \alpha x n)^{\lambda}}\right] d x \leq\|f\|_{p, \Phi} L .
$$

Then by (15), we have (13). On the other-hand, assuming that (13) is valid, setting

$$
f(x):=[\Phi(x)]^{1-q}\left[\sum_{n=2}^{\infty} \frac{a_{n}}{(\ln \alpha x n)^{\lambda}}\right]^{q-1}, \quad x \in\left(\frac{1}{\sqrt{\alpha}}, \infty\right),
$$

then $L^{q-1}=\|f\|_{p}, \Phi$. . By (12), we find $L<\alpha$. If $L=0$, then (15) is valid trivially; if $L$ $>0$, then by (13), we have

$$
\begin{aligned}
& \|f\|_{p, \Phi}^{p}=L^{q}=I<B\left(\lambda_{1}, \lambda_{2}\right)\|f\|_{p, \Phi}\|a\|_{q, \Psi}, \text { i.e. } \\
& \|f\|_{p, \Phi}^{p-1}=L<B\left(\lambda_{1}, \lambda_{2}\right)\|a\|_{q, \Psi},
\end{aligned}
$$

That is, (15) is equivalent to (13). Hence inequalities (13), (14) and (15) are equivalent.

For $0<\varepsilon<p \lambda_{1}$, setting $\tilde{a}_{n}=\frac{1}{n}(\ln \sqrt{\alpha} n)^{\lambda_{2}-\frac{\epsilon}{q}-1}, n \in \mathbf{N} \backslash\{1\}$, and

$$
\tilde{f}(x):=\left\{\begin{array}{c}
0, x \in\left(\frac{1}{\sqrt{\alpha}}, \frac{e}{\sqrt{\alpha}}\right) \\
\frac{1}{x}(\ln \sqrt{\alpha} x)^{\lambda_{1}-\frac{\epsilon}{p}-1}, \quad x \in\left[\frac{e}{\sqrt{\alpha}}, \infty\right)
\end{array},\right.
$$

if there exists a positive number $k\left(\leq B\left(\lambda_{1}, \lambda_{2}\right)\right)$, such that (13) is valid as we replace $B$ $\left(\lambda_{1}, \lambda_{2}\right)$ with $k$, then in particular, it follows

$$
\begin{aligned}
\tilde{I}: & =\sum_{n=2}^{\infty} \int_{\frac{1}{\sqrt{\alpha}}}^{\infty} \frac{1}{(\ln \alpha x n)^{\lambda}} \tilde{a}_{n} \tilde{f}(x) d x<k\|\tilde{f}\|_{p, \Phi}\|\tilde{a}\|_{q, \Psi} \\
& =k\left\{\int_{\frac{e}{\sqrt{\alpha}}}^{\infty} \frac{d x}{x(\ln \sqrt{\alpha} x)^{\varepsilon+1}}\right\}^{\frac{1}{p}}\left\{\frac{1}{2(\ln 2 \sqrt{\alpha})^{\varepsilon+1}}+\sum_{n=3}^{\infty} \frac{1}{n(\ln \sqrt{\alpha} n)^{\varepsilon+1}}\right\}^{\frac{1}{q}} \\
& <k\left(\frac{1}{\varepsilon}\right)^{\frac{1}{p}}\left\{\frac{1}{2(\ln 2 \sqrt{\alpha})^{\varepsilon+1}}+\int_{2}^{\infty} \frac{1}{x(\ln \sqrt{\alpha} x)^{\varepsilon+1}} d x\right\}^{\frac{1}{q}} \\
& =\frac{k}{\varepsilon}\left\{\frac{\varepsilon}{2(\ln 2 \sqrt{\alpha})^{\varepsilon+1}}+\frac{1}{(\ln 2 \sqrt{\alpha})^{\varepsilon}}\right\}^{\frac{1}{q}},
\end{aligned}
$$




$$
\begin{gathered}
\tilde{I}=\sum_{n=2}^{\infty}(\ln \sqrt{\alpha} n)^{\lambda_{2}-\frac{\varepsilon}{q}-1} \frac{1}{n} \int_{\frac{e}{\sqrt{\alpha}}}^{\infty} \frac{1}{x(\ln \alpha x n)^{\lambda}}(\ln \sqrt{\alpha} x)^{\lambda_{1}-\frac{\varepsilon}{p}-1} d x \\
t=(\ln \sqrt{\alpha} x) /(\ln \sqrt{\alpha} n) \sum_{n=2}^{\infty} \frac{1}{n(\ln \sqrt{\alpha} n)^{\varepsilon+1}} \int_{1 / \ln \sqrt{\alpha} n}^{\infty} \frac{t t^{\lambda_{1}-\frac{\varepsilon}{p}-1}}{(t+1)^{\lambda}} d t \\
=B\left(\lambda_{1}-\frac{\varepsilon}{p}, \lambda_{2}+\frac{\varepsilon}{p}\right) \sum_{n=2}^{\infty} \frac{1}{n(\ln \sqrt{\alpha} n)^{\varepsilon+1}}-A(\varepsilon) \\
>B\left(\lambda_{1}-\frac{\varepsilon}{p}, \lambda_{2}+\frac{\varepsilon}{p}\right) \int_{2}^{\infty} \frac{1}{y(\ln \sqrt{\alpha} y)^{\varepsilon+1}} d y-A(\varepsilon) \\
=\frac{1}{\varepsilon(\ln 2 \sqrt{\alpha})^{\varepsilon}} B\left(\lambda_{1}-\frac{\varepsilon}{p}, \lambda_{2}+\frac{\varepsilon}{p}\right)-A(\varepsilon), \\
A(\varepsilon):=\sum_{n=2}^{\infty} \frac{1}{n(\ln \sqrt{\alpha} n)^{\varepsilon+1}} \int_{0}^{1 / \ln \sqrt{\alpha} n} \frac{1}{(t+1)^{\lambda}} t^{\lambda_{1}-\frac{\varepsilon}{p}-1} d t .
\end{gathered}
$$

W find

$$
\begin{aligned}
& 0<A(\varepsilon) \leq \sum_{n=2}^{\infty} \frac{1}{n(\ln \sqrt{\alpha} n)^{\varepsilon+1}} \int_{0}^{1 / \ln \sqrt{\alpha} n} t^{\lambda_{1}-\frac{\varepsilon}{p}-1} d t \\
& =\frac{1}{\lambda_{1}-\frac{\varepsilon}{p}} \sum_{n=2}^{\infty} \frac{1}{n(\ln \sqrt{\alpha} n)^{\lambda_{1}+\frac{\varepsilon}{q}+1}}<\infty \text {, }
\end{aligned}
$$

that is, $A(\varepsilon)=O(1)\left(\varepsilon \rightarrow 0^{+}\right)$. Hence by (18) and (19), it follows

$$
\frac{B\left(\lambda_{1}-\frac{\varepsilon}{p}, \lambda_{2}+\frac{\varepsilon}{p}\right)}{(\ln 2 \sqrt{\alpha})^{\varepsilon}}-\varepsilon O(1)<k\left\{\frac{\varepsilon}{2(\ln 2 \sqrt{\alpha})^{\varepsilon+1}}+\frac{1}{(\ln 2 \sqrt{\alpha})^{\varepsilon}}\right\}^{\frac{1}{q}}
$$

and $B\left(\lambda_{1}, \lambda_{2}\right) \leq k\left(\varepsilon \rightarrow 0^{+}\right)$. Hence, $k=B\left(\lambda_{1}, \lambda_{2}\right)$ is the best value of (13).

Due to the equivalence, the constant factor $B\left(\lambda_{1}, \lambda_{2}\right)$ in (14) and (15) is the best possible. Otherwise, we can imply a contradiction by (16) and (17) that the constant factor in (13) is not the best possible. $\square$ •

Remark 1 (i) Define the first type half-discrete Mulholland's operator $T: L_{p, \Phi}\left(\frac{1}{\sqrt{\alpha}}, \infty\right) \rightarrow l_{p, \Psi 1-p a s}$ follows: for $f \in L_{p, \Phi}\left(\frac{1}{\sqrt{\alpha}}, \infty\right)$, we define $T f \in l_{p, \Psi 1-p a s}$

$$
T f(n)=\int_{\frac{1}{\sqrt{\alpha}}}^{\infty} \frac{1}{(\ln \alpha x n)^{\lambda}} f(x) d x, \quad n \in \mathbf{N} \backslash\{1\} .
$$

Then by (14), it follows $\|T f\|_{p, \Psi^{1-p}} \leq B\left(\lambda_{1}, \lambda_{2}\right)\|f\|_{p, \Phi}$ and then $T$ is a bounded operator with $\|T\| \leq B\left(\lambda_{1}, \lambda_{2}\right)$. Since by Theorem 1 , the constant factor in (14) is the best possible, we have $\|T\|=B\left(\lambda_{1}, \lambda_{2}\right)$. 
(ii) Define the second type half-discrete Mulholland's operator $\tilde{T}: l_{q, \Psi} \rightarrow L_{q, \Phi^{1-q}}\left(\frac{1}{\sqrt{\alpha}}, \infty\right)$ as follows: For a $L l_{q, \psi}$, define $\tilde{T} a \in L_{q, \Phi^{1-q}}\left(\frac{1}{\sqrt{\alpha}}, \infty\right)$ as

$$
\tilde{T} a(x)=\sum_{n=2}^{\infty} \frac{1}{(\ln \alpha x n)^{\lambda}} a_{n}, \quad x \in\left(\frac{1}{\sqrt{\alpha}}, \infty\right) .
$$

Then by (15), it follows $\|\tilde{T} a\|_{q . \Phi^{1-q}} \leq B\left(\lambda_{1}, \lambda_{2}\right)\|a\|_{q, \Psi \text { and }}$ then $\tilde{T}$ is a bounded operator with $\|\tilde{T}\| \leq B\left(\lambda_{1}, \lambda_{2}\right)$. Since by Theorem 1 , the constant factor in (15) is the best possible, we have $\|\tilde{T}\|=B\left(\lambda_{1}, \lambda_{2}\right)$.

Remark 2 We set $p=q=2, \lambda=1, \lambda_{1}=\lambda_{2}=\frac{1}{2}$ in (13), (14) and (15). (i) if $\alpha=\frac{4}{9}$, then we deduce (7) and the following equivalent inequalities:

$$
\begin{aligned}
& \sum_{n=2}^{\infty} \frac{1}{n}\left[\int_{\frac{3}{2}}^{\infty} \frac{f(x)}{\ln \frac{4}{9} x n} d x\right]^{2}<\pi^{2} \int_{\frac{3}{2}}^{\infty} x f^{2}(x) d x, \\
& \int_{\frac{3}{2}}^{\infty} \frac{1}{x}\left[\sum_{n=2}^{\infty} \frac{a_{n}}{\ln \frac{4}{9} x n}\right]^{2} d x<\pi^{2} \sum_{n=2}^{\infty} n a_{n^{2}}^{2}
\end{aligned}
$$

(ii) if $\alpha=1$, then we have the following half-discrete Mulholland's inequality and its equivalent forms:

$$
\begin{aligned}
& \int_{1}^{\infty} f(x) \sum_{n=2}^{\infty} \frac{a_{n}}{\ln x n} d x<\pi\left\{\int_{1}^{\infty} x f^{2}(x) d x \sum_{n=2}^{\infty} n a_{n}^{2}\right\}^{\frac{1}{2}}, \\
& \sum_{n=2}^{\infty} \frac{1}{n}\left[\int_{1}^{\infty} \frac{f(x)}{\ln x n} d x\right]^{2}<\pi^{2} \int_{1}^{\infty} x f^{2}(x) d x, \\
& \int_{1}^{\infty} \frac{1}{x}\left[\sum_{n=2}^{\infty} \frac{a_{n}}{\ln x n}\right]^{2} d x<\pi^{2} \sum_{n=2}^{\infty} n a_{n}^{2} .
\end{aligned}
$$

\section{Acknowledgements}

This work is supported by the Guangdong Science and Technology Plan Item (No. 2010B010600018), and the Guangdong Modern Information Service industry Develop Particularly item 2011 (No. 13090).

\section{Author details}

${ }^{1}$ Department of Computer Science, Guangdong University of Education, Guangzhou, Guangdong 510303, PR China

${ }^{2}$ Department of Mathematics, Guangdong University of Education, Guangzhou, Guangdong 510303, PR China

\section{Authors' contributions}

QC conceived of the study, and participated in its design and coordination. BY wrote and reformed the article. All authors read and approved the final manuscript.

\section{Competing interests}

The authors declare that they have no competing interests. 


\section{References}

1. Hardy, GH, Littlewood, JE, Pólya, G: Inequalities. Cambridge University Press, Cambridge (1934)

2. Mitrinović, DS, Pečarić, JE, Fink, AM: Inequalities Involving Functions and Their Integrals and Derivatives. Kluwer Acaremic Publishers, Boston (1991)

3. Yang, B: Hilbert-Type Integral Inequalities. Bentham Science Publishers Ltd., dubai (2009)

4. Yang, B: Discrete Hilbert-Type Inequalities. Bentham Science Publishers Ltd., dubai (2011)

5. Yang, B: An extension of Mulholand's inequality. Jordan J Math Stat. 3(3):151-157 (2010)

6. Yang, B: On Hilbert's integral inequality. J Math Anal Appl. 220, 778-785 (1998)

7. Yang, B: The Norm of Operator and Hilbert-type Inequalities. Science Press, Beijin (2009)

8. Yang, B, Brnetić, I, Krnić, M, Pečarić, J: Generalization of Hilbert and Hardy-Hilbert integral inequalities. Math Inequal Appl. 8(2):259-272 (2005)

9. Krnić, M, Pečarić, J: Hilbert's inequalities and their reverses. Publ Math Debrecen. 67(3-4):315-331 (2005)

10. Jin, J, Debnath, L: On a Hilbert-type linear series operator and its applications. J Math Anal Appl. 371, 691-704 (2010)

11. Azar, L: On some extensions of Hardy-Hilbert's inequality and applications. J Inequal Appl 2009, 14 (2009). Article ID 546829

12. Yang, B, Rassias, T: On the way of weight coefficient and research for Hilbert-type inequalities. Math Inequal Appl. 6(4):625-658 (2003)

13. Arpad, B, Choonghong, O: Best constant for certain multilinear integral operator. J Inequal Appl 2006, 12 (2006). Article ID 28582

14. Kuang, J, Debnath, L: On Hilbert's type inequalities on the weighted Orlicz spaces. Pac J Appl Math. 1(1):95-103 (2007)

15. Zhong, W: The Hilbert-type integral inequality with a homogeneous kernel of Lambda-degree. J Inequal Appl 2008, 13 (2008). Article ID 917392

16. Li, Y, He, B: On inequalities of Hilbert's type. Bull Aust Math Soc. 76(1):1-13 (2007)

17. Yang, B: A mixed Hilbert-type inequality with a best constant factor. Int J Pure Appl Math. 20(3):319-328 (2005)

18. Yang, B: A half-discrete Hilbert's inequality. J Guangdong Univ Edu. 31(3):1-7 (2011)

19. Donald, LC: Measure Theorey. Birkhäuser, Boston (1980)

doi:10.1186/1029-242X-2012-70

Cite this article as: Chen and Yang: On a more accurate half-discrete mulholland's inequality and an extension.

Journal of Inequalities and Applications 2012 2012:70.

\section{Submit your manuscript to a SpringerOpen ${ }^{\circ}$ journal and benefit from:}

- Convenient online submission

- Rigorous peer review

- Immediate publication on acceptance

- Open access: articles freely available online

- High visibility within the field

- Retaining the copyright to your article

Submit your next manuscript at $>$ springeropen.com 